\title{
The Early Bonobo Gets the Juice? The Evolutionary Roots of Pre-crastination in Bonobos (Pan paniscus)
}

\author{
Natalie Schwob ${ }^{1, *}$, Amanda J. Epping ${ }^{2}$, Jared P. Taglialatela ${ }^{2,3}$, and Daniel J. Weiss ${ }^{1,4}$ \\ ${ }^{1}$ The Pennsylvania State University, Department of Psychology, University Park, PA, USA \\ ${ }^{2}$ Ape Cognition and Conservation Initiative, Des Moines, IA, USA \\ ${ }^{3}$ Kennesaw State University, Department of Ecology, Evolution and Organismal Biology, Kennesaw, GA, USA \\ ${ }^{4}$ The Pennsylvania State University, Program in Linguistics, University Park, PA, USA \\ *Corresponding author (Email: ngschwob@gmail.com)
}

Citation - Schwob, N., Epping, A. J., Taglialatela, J. P., \& Weiss, D. J. (2022). The early bonobo gets the juice? The evolutionary roots of pre-crastination in bonobos (Pan paniscus). Animal Behavior and Cognition, 9(1), 3-13. https://doi.org/10.26451/abc.09.01.02.2022

\begin{abstract}
Pre-crastination refers to the propensity to initiate tasks at the earliest possible moment. Research with human adults has found that some individuals consistently chose to transport a nearby object a further distance rather than delay initiation of the transport to select an object closer to the target. This phenomenon has never been tested in animals using analogous methods. Consequently, we tested bonobos - the species most closely related to humans using two versions of a comparable transport task. Overall, we found that all five bonobos tended to select the first object they encountered to transport to the goal. Unlike humans, the bonobos sometimes transported both available objects. Two of the five bonobos consistently pre-crastinated, a similar proportion to that found in human experiments. However, if the pre-crastination choice was non-functional, the bonobos chose the motorically efficient choice. In sum, our findings provide an existence proof for pre-crastination tendencies in some bonobos, akin to the distribution of this trait in humans. We discuss the possibility that the pre-crastination choice represents an automatic response triggered by the affordances of the objects encountered.
\end{abstract}

Keywords - Action planning, Primates, Decision making, Pre-crastination

One of the cornerstones of decision-making theories concerns minimizing effort (Kahneman \& Tversyky, 1979). Several prominent theories, such as the law of less work (Hull, 1943) and the principle of least effort (Zipf, 1949), suggest that when individuals choose between alternative actions, they inevitably select "the less laborious behavior sequence" (Hull, 1943; p. 249). In that context, the discovery that some individuals pre-crastinate may be both surprising and counterintuitive. Pre-crastination is the tendency to initiate actions at the earliest possible moment in order to hasten subgoal completion, even at the expense of exerting greater physical effort (Fournier et al., 2019; Rosenbaum et al., 2014).

The term pre-crastination was first coined in a study with human adults who were tasked with transporting one of two buckets placed at different distances from a target location. To the surprise of the researchers, the majority of participants selected the bucket closest to the starting point, despite having to transport it a farther distance relative to the bucket placed closer to the target location (Rosenbaum et al., 2014). The authors surmised that pre-crastination served to reduce the cognitive demands of the task by initiating an action at the earliest possible moment (Rosenbaum et al., 2019). Notably, the tendency to precrastinate is not ubiquitous. For example, a subsequent study found that $29 \%$ of participants consistently pre-crastinated, while $45 \%$ consistently preferred the motorically efficient choice (with the remainder dithering between choices for reasons that were unclear; Raghunath et al., 2020). 
The present study explores the evolutionary roots of pre-crastination, assessing whether the tendency to initiate actions at the earliest possible moment is also present in some members of our closest primate relatives, bonobos (Pan paniscus). We sought to test the hypothesis that some bonobos precrastinate. To the best of our knowledge, this study represents the first test of any nonhuman using an analogous method as the studies with humans.

There have been very few studies of pre-crastination in nonhuman species, and these have been conducted with pigeons (Columba livia). The design of these studies has drastically differed from human studies in terms of training and task demands. One of the tasks (Wasserman \& Brzykcy, 2015) began with a single, colored square presented in the center of the screen. When the pigeon pecked the square, it disappeared for half a second and reappeared with an additional adjacent square flanking it on either left or right, depending on the color of the target. Either of the two squares (middle or flanker) could then be pecked to elicit a third and final square that appeared on the same side as the flanker square. The pigeons received a food reward for pecking this final square irrespective of the location of the second peck. The pigeons selected the flanker square for the second peck more often than the center square. Wasserman and Brzykcy (2015) considered this pre-crastination as the pigeons moved to the final position earlier than necessary, as pecking either the flanker or center square would have progressed them to the reward stage.

Notably, the paradigm used with pigeons differed along several critical dimensions from the original human study conducted by Rosenbaum and colleagues (2014). Zentall et al. (2018) suggested that pecking on the side square would bring the pigeon into closer proximity of the reward square, and thus confer some advantage. This notion also seems consistent with the literature on motor planning in humans that finds reaction time savings for repeated movements (e.g., Lebkuecher et al., in review; Valyear et al., 2019). Moreover, unlike the studies conducted with humans, it is possible that executing the action early might produce a benefit with regard to priming the final response (and similarly, the anticipation of the side of the reward might have primed the selection of the flanker square). Critically, the cost for the pigeons making the flanker selection is unclear, and thus it is unknown whether this anticipatory behavior would occur in circumstances that resulted in greater physical effort.

With some of these issues in mind, Zentall et al. (2018) tested pigeons with a method that approximated different aspects of the Rosenbaum et al. (2014) pre-crastination study. They presented pigeons with a choice between two sequences of events that were equated with respect to overall time and reward. One chain started with an initial peck that would lead to a $15 \mathrm{~s}$ delay, followed by a presentation of a key that, when pecked, resulted in reinforcement following a $5 \mathrm{~s}$ delay. The other option reversed the contingency with an initial delay of $5 \mathrm{~s}$ and a subsequent $15 \mathrm{~s}$ delay until reinforcement. The authors surmised that pre-crastination would be evidenced if the pigeons selected the $5 \mathrm{~s}$ delay first (thereby reaching second peck sooner). However, the pigeons overwhelmingly preferred the $15 \mathrm{~s}$ and then $5 \mathrm{~s}$ chain, and thus were considered to have procrastinated by choosing to temporally delay the first peck, rather than executing the action earlier (Zentall et al., 2018). We note that similarly to the Wasserman and Brzykcy (2015) study, this task also imposed no additional physical cost associated with the earlier choice. Irrespective of the differences between these tasks and the bucket task used with humans, the evidence for pre-crastination in any nonhuman species can be considered mixed at best, and this is perhaps related to the timing of the reward delivery relative to the actions taken by the animals (see Rosenbaum et al., 2019, for further discussion).

Unlike the pigeon studies that involved training and lacked motor costs, we utilized methods that more closely approximate those employed by human studies. To our knowledge, this is also the first study of pre-crastination with nonhuman primates. Finding a homologous tendency in bonobos can provide insight as to whether the phylogenetic roots of action-planning heuristics were shared with the last common ancestor of humans and other apes. In Experiment 1a, bonobos selected between two identical tools that could be carried to a target location in order to extract a food reward. The tools were placed at different distances from the entry point for the bonobos, and accordingly, different distances from the target. In Experiment $1 \mathrm{~b}$, one of the tools was replaced by a non-functional object with similar visual properties to examine the possibility that the bonobos had a tendency to pick up all objects in sight. In Experiment 1c, cups were used in place of the tools in effort to make the task requirements more apparent to all participants. 
The cups could be brought to an experimenter who would provision a juice reward. Similar to the human experiments, we considered the selection of the object closest to the starting point, and therefore furthest from the target, as the choice that would indicate pre-crastination.

\section{Method}

\section{Ethics Statement}

No animals were forced to participate, nor were there any injuries or stress related to participation in the study. The study was approved by the Institutional Animal Care and Use Committee at the Ape Cognition and Conservation Initiative. Experimental procedures conformed to the regulations of the IACUCs at the Ape Initiative and The Pennsylvania State University.

\section{Participants}

We tested five bonobos ( 1 female; 8 - 39 years old; see Table 1 ) at the Ape Cognition and Conservation Initiative (Ape Initiative) in Des Moines, Iowa. Bonobos were tested individually and voluntarily participated. All animals have a history of participating in research tasks and were housed in accordance with guidelines provided by the USDA and the Association of Zoos \& Aquariums.

Table 1

Bonobo Demographic Information at the Onset of Data Collection

\begin{tabular}{lcc}
\hline Bonobo & Sex & Age \\
\hline Kanzi & Male & 39 \\
Elikya & Female & 22 \\
Nyota & Male & 21 \\
Maisha & Male & 19 \\
Teco & Male & 8 \\
\hline
\end{tabular}

\section{Stimuli}

In Phase 1, bonobos were tested in an empty room $(5.5 \mathrm{~m} \times 8.5 \mathrm{~m})$ except for two $30.5 \mathrm{~cm}$ white lollipop sticks placed on the ground in the center of a $30.5 \mathrm{~cm}$ x $30.5 \mathrm{~cm}$ square outlined with brightly colored chalk (see Figure 1). All objects across all three experiments were placed in the center of pink chalk squares that were meant to increase visibility. A target PVC pipe-feeder was filled with yogurt or mustard, both highly valued foods (the choice of reward was dictated by the individual's calorie allotment left for the day). The pipe-feeder was attached to the exterior of the enclosure $5.5 \mathrm{~m}$ from the testing room entrance. All bonobos were familiar with using sticks as tools to extract food. In Phase 2, the tool closest to the entrance was replaced with a non-functional object that shared similar features; either a $2.54 \mathrm{~cm} \mathrm{x}$ $30.5 \mathrm{~cm}$ strip of white paper or a $2.54 \mathrm{~cm}$ x $30.5 \mathrm{~cm}$ strip of folded duct tape - these objects were similar in appearance to the functional tool but lack the rigidity to be used as a tool. Phase 3 was completed in a $8.5 \mathrm{~m}$ x $5.2 \mathrm{~m}$ room that was empty except for two cups $(16.8 \mathrm{~cm} \mathrm{~h}$ x $8.9 \mathrm{~cm}$ diameter). An experimenter sat $6 \mathrm{~m}$ from the entrance behind a mesh barrier with a bottle of juice. All bonobos were familiar with cups and receiving juice rewards. 
Figure 1

A Schematic for the Setup of Each Study

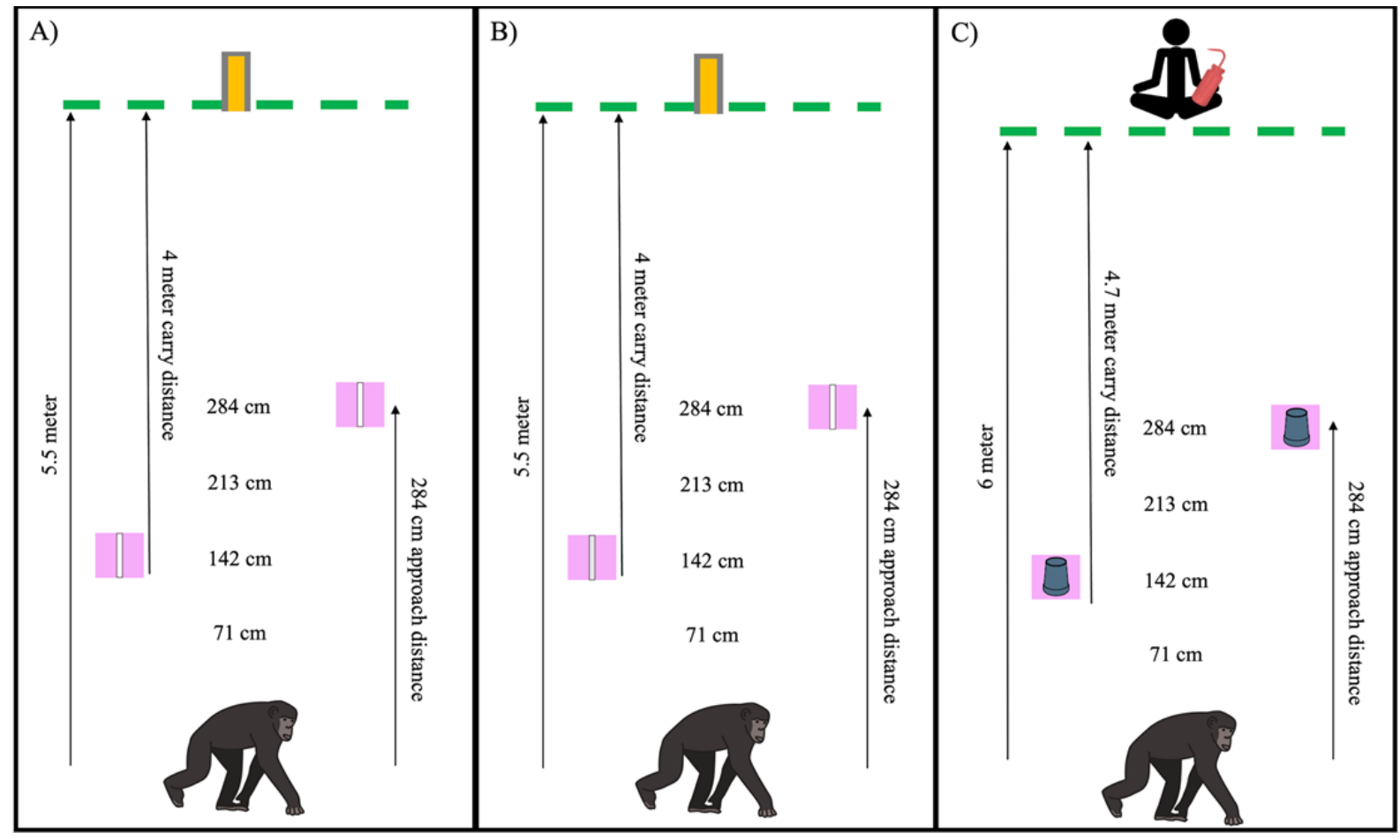

Note. A) Phase 1. B) Phase 2, where the thin gray rectangle represents the non-functional object. C) Phase 3

\section{Design and Procedure}

\section{Phase 1}

Subjects entered the testing room and selected an object to transport to the pipe-feeder. Bonobos performed a minimum of five familiarization trials prior to testing in which a single tool was placed 30 $\mathrm{cm}$ directly in front of the target in a chalk square. The goal of the familiarization trials was to associate the chalk square with the presence of a tool. Doing so would allow the bonobos to look into the testing room prior to the onset of trials and appreciate the number and location of possible tools. Successful familiarization required bonobos to promptly select the tool and extract the reward three times in a row. If the bonobo did not move directly towards the object, did not pick up the tool before engaging with the target, or picked up the object and walked away from the target, the familiarization trial was not considered valid (and was subsequently rerun).

Testing trials were modeled on the original human study (Rosenbaum et al., 2014) with two tools placed at equidistant locations to the left and right of the (unmarked) direct line extending between the entrance to the room and the target (see Figure 1). The tools were placed at distances of either $71 \mathrm{~cm}, 142$ $\mathrm{cm}, 213 \mathrm{~cm}$, or $284 \mathrm{~cm}$ from the entrance depending on the trial. There were 12 possible unequal location combinations (e.g., $71 \mathrm{~cm}$ on the right, $284 \mathrm{~cm}$ on the left), with no trials using equivalent approach distances (e.g., $142 \mathrm{~cm}$ on both the right and left). This design led to 12 trials with the short approach distance tool on the right and 12 trials with the short approach distance tool on the left. Given the size of the tools, the bonobos could select either object or both (sequentially). Each asymmetric distance combination was completed twice for each subject, with combinations quasi-randomized such that no approach distance was repeated on the same side in successive trials, as was true for all phases. 
To begin testing, the experimenter placed the two tools at their pre-determined locations in chalk squares while the bonobo waited outside the testing room. The experimenter allowed entry only after the bonobo gazed toward the experimenter. In all phases, apes were rewarded regardless of object selection. All trials were video recorded and subsequently coded by an experimenter and a research assistant blind to the hypotheses. The coders rated object selection with 100\% inter-coder reliability (in all experimental conditions).

\section{Phase 2}

After 24 hours elapsed following the last trial of Phase 1, the bonobos completed one trial of each distance combination for Phase 2. The setup was identical to Phase 1 except that the object with the short approach distance was a non-functional tool while the object with the long approach distance was the same functional tool used in Phase 1. Prior to this experiment, the bonobos were given 15 minutes to become familiar with the non-functional tools to ensure they would not select the item due to its novelty. Bonobos then completed six trials per non-functional object with no more than five trials in a single day.

\section{Phase 3}

The procedure for Phase 3 was identical to Phase 1 except for the use of cups rather than sticks and juice as the reward rather than mustard or yogurt. All five bonobos had five familiarization trials that consisted of a single cup placed in the center of a brightly colored chalk square $5.8 \mathrm{~m}$ from the room entrance, and $0.3 \mathrm{~m}$ from the experimenter. In order to pass familiarization, subjects had to enter the room, grasp the cup, and transport it directly to the researcher to receive a juice reward. All five bonobos successfully passed all five familiarization trials and subsequently began testing trials within the next 24 hours. Each distance combination $(71 \mathrm{~cm}, 142 \mathrm{~cm}, 213 \mathrm{~cm}$, or $284 \mathrm{~cm}$ from the entrance) was presented once resulting in 12 trials per ape, with six trials with the short approach distance cup at the ape's right and six trials with the short approach distance cup at the left (randomly distributed).

\section{Results}

\section{Phase 1}

In Phase 1, only two bonobos passed their first five familiarization trials. The other three bonobos neglected the tool and showed minimal interest in participating after approximately 15 familiarization trials. Consequently, these individuals were excluded from Phase 1 and Phase 2 and we created a parallel task (Phase 3) to encourage greater participation. The two bonobos in Phase 1 completed 24 trials each, yielding a total of 48 trials. Of these 48 trials, the object with the short approach distance was transported 43 times (see Table 2 and Figure 2). One bonobo transported both objects in 16 of these trials. We used a binomial test ( $\mathrm{R}$ version 4.0.0; $\mathrm{R}$ Core Team, 2016) with chance at $25 \%$ as random behavior could generate four outcomes: transporting the object with the short approach distance, transporting the object with the long approach distance, transporting both or neither of the objects. We found both bonobos transported the first object they approached more than would be expected by chance, with Kanzi choosing the first object alone more often than chance $(87.50 \%, p<.001,95 \% \mathrm{CI}[0.68,0.97])$ and Teco choosing both objects more often than chance $(66.67 \%, p<.001,95 \%$ CI $[0.45,0.84])$. 
Table 2

Raw Data of the Results of All Phases

\begin{tabular}{|c|c|c|c|c|c|}
\hline Experiment & Ape & $\begin{array}{l}\text { Short Approach } \\
(N)\end{array}$ & Both $(N)$ & $\begin{array}{l}\text { Long Approach } \\
(N)\end{array}$ & Trials $(N)$ \\
\hline \multirow{3}{*}{ Phase 1: Tools } & Kanzi & 21 & 0 & 3 & 24 \\
\hline & Teco & 6 & 16 & 2 & 24 \\
\hline & Overall & 27 & 16 & 5 & 48 \\
\hline \multirow{3}{*}{$\begin{array}{c}\text { Phase 2: } \\
\text { Nonfunctional }\end{array}$} & Kanzi & 0 & 0 & 12 & 12 \\
\hline & Teco & 0 & 1 & 11 & 12 \\
\hline & Overall & $\mathbf{0}$ & 1 & 23 & 24 \\
\hline \multirow{6}{*}{ Phase 3: Cups } & Kanzi & 11 & 0 & 1 & 12 \\
\hline & Elikya & 8 & 1 & 2 & 11 \\
\hline & Maisha & 2 & 8 & 2 & 12 \\
\hline & Nyota & 6 & 3 & 3 & 12 \\
\hline & Teco & 0 & 8 & 0 & 8 \\
\hline & Overall & 27 & 20 & 8 & 55 \\
\hline
\end{tabular}

Phase 2

Both bonobos successfully completed 12 trials each. Across both subjects, the nonfunctional object with the short approach distance was selected once out of 24 trials. Both apes ignored the nonfunctional object with the short approach distance and transported the functional object with the long approach distance more often than would be expected by chance (Kanzi: $0 \%, p<.001,95 \%$ CI $[0.74$, 1.00]; Teco: $8.33 \%, p<.001,95 \%$ CI $[0.62,0.99])$. For the single trial in which the non-functional object was selected, it was not transported. Using a z-test of proportions, we found that the proportion of transporting the object with the short approach distance was significantly higher in Phase 1 than in Phase $2(z=6.75, p<.001,95 \%$ CI $[0.724,1.000])$.

\section{Phase 3}

All five bonobos passed their first five familiarization trials and completed Phase 3. Four bonobos completed all 12 trials and one completed only 11 trials. The juvenile bonobo (Teco) completed all 12 trials; however, on four of these he did not select a cup while moving towards the target, and thus these four trials were not included in the analyses. Maisha and Nyota did not select a tool in 3 and 4 trials respectively. Consequently, these trials were re-run.

Of the five bonobos and 55 trials included in this analysis, the cup with the short approach distance was transported 47 times $(85.45 \%)$. Of these 47 trials, both cups were transported 20 times $(42.55 \%$ which was equivalent to $36.36 \%$ out of all 55 trials). The cup with the long approach distance was exclusively transported eight time across all 55 trials (14.55\%). Binomial tests were completed for each individual with chance set again at $25 \%$. Two of the five bonobos were more likely than chance to transport the cup with the short approach distance alone (Kanzi: $91.67 \%, p<.001,95 \% \mathrm{CI}[0.42,1.00]$; Elikya: $72.73 \%, p$ $=.001,95 \%$ CI $[0.39,0.74])$. Of the remaining three bonobos, two transported both objects at greater than chance levels (Maisha: $66.67 \%, p=.003,95 \%$ CI [0.35, 0.90]; Teco: $100 \%, p=.008,95 \%$ CI [0.63, 1.0]). The final bonobo trended towards significance for selecting the object with the short approach distance alone (Nyota: $50 \%, p=.086,95 \%$ CI [0.21, 0.79]). This bonobo also transported the cup on the right 11 of 12 trials, suggesting a possible side bias. 
Figure 2

Object Selection for Phase 1, Phase 2, and Phase 3 for Each Bonobo

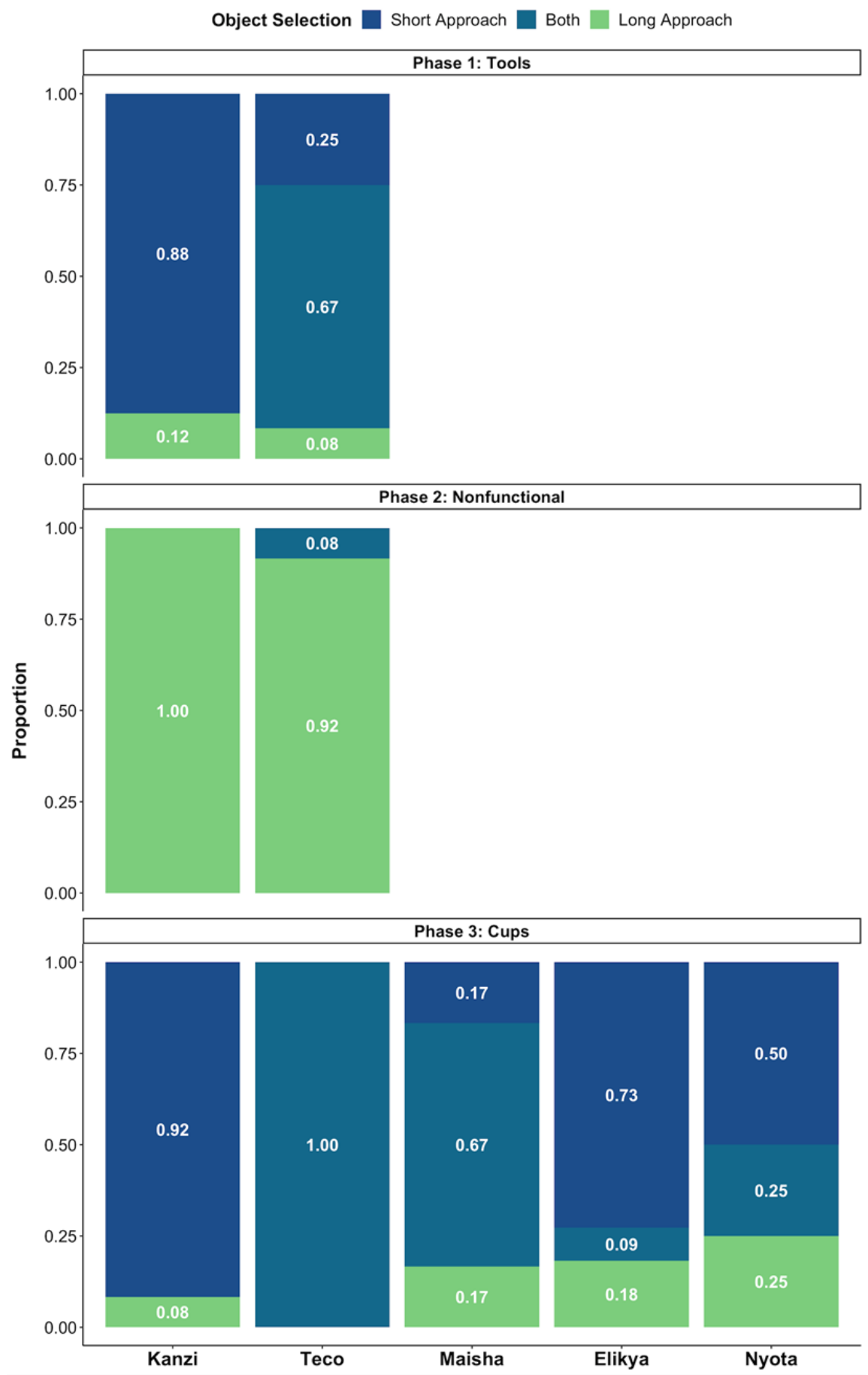

Discussion

Pre-crastination is the tendency to initiate a task as soon as possible, an effect observed even when early initiation incurs additional physical cost (Fournier et al., 2019; Rosenbaum et al., 2014). This study explored whether pre-crastination behaviors exist in bonobos in the context of a transport task. In 
Phase 1, we presented bonobos with two sticks with which they could extract a food reward. One stick was nearer to the subject and farther from the goal (i.e., short approach and longer transport distance), and one was farther from the subject and closer to the goal (i.e., long approach and shorter transport distance), similar to human studies of this behavior (e.g., Rosenbaum et al., 2014). However, only two bonobos passed the familiarization trials and progressed to this test. Of these two bonobos, one consistently selected the tool with the short approach distance even though it resulted in a longer transport. The other bonobo frequently selected the first tool as well, but often transported both tools to the goal, which was not anticipated. In Phase 2, the short approach tool was replaced by a non-functional object and both bonobos consistently selected only the functional tool that had the longer approach distance. Phase 3 was designed to facilitate the participation of more subjects. The bonobos were presented with two cups that could be transported to an experimenter who then would provide a juice reward. Consistent with the other phases of the experiment, one cup was nearer to the subject and required a longer transport distance and one was further from the subject but required a shorter transport distance. All five bonobos completed the experimental task. We found that two of the five bonobos transported the object with the short approach distance and ignored the object closest to the goal (i.e., pre-crastinated). A third bonobo trended in this direction as well but did not reach significance. The remaining two bonobos selected both cups at greater than chance levels. While there was considerable variability between selecting either the first cup or both cups, the fact that at least two individuals very consistently chose the first cup provides an existence proof that pre-crastination, as it has been defined in the human literature (Rosenbaum et al., 2014), can also be found among members of the closest living relatives to humans.

Despite the variability noted above, the proportion of pre-crastinators in our study was actually quite comparable to that reported in human research (Raghunath et al., 2020). Studies reporting individual differences in pre-crastination in humans have reported that $29 \%-40 \%$ of participants consistently precrastinate (Blinch \& DeWinne, 2019; Raghunanth et al., 2020; see also Fournier et al., 2019). Similarly, in Phase 3, we found that $40 \%$ of our sample consistently pre-crastinated, exhibiting a statistically significant preference for transporting only the object with the short approach distance. While we acknowledge our sample size was small (especially relative to the human studies), access to bonobos living in AZA-accredited facilities in which these types of experiments can be conducted is extraordinarily limited. Nevertheless, one salient difference across species is that the human studies found between $40-45 \%$ of participants rarely pre-crastinated, preferring the motorically efficient choice (Blinch \& DeWinne, 2019; Raghunanth et al., 2020). This behavior was not evident in any of the individuals in our study. Rather, all five bonobos exhibited a preference for transporting the firstencountered object with the short approach distance, though several bonobos transported the second object as well. Selecting both objects was also a notable departure from human participants (Rosenbaum et al., 2014) who followed the explicit instructions provided by the experimenters to select a single bucket for transport. We note that despite transporting two objects, the bonobos never attempted to obtain an additional reward by presenting the second object, nor did they appear frustrated in receiving a single reward. The results of Phase 2 suggest that it was possible, at least in principle, for an individual prone to selecting two objects to forgo the selection of the first encountered object.

While these findings suggest evolutionary continuity in pre-crastination among some individuals, a related question is whether these behaviors arise due to similar cognitive processes. This issue has not been fully resolved in humans (Rosenbaum \& Wasserman, 2015), so correspondingly it would be difficult to make any claims regarding shared cognitive features. The initial study (Rosenbaum et al., 2014) suggested that selecting the first encountered object hastened the completion of the subgoal and thereby reduced the cognitive load associated with the task. However, we note that several follow-up studies have suggested that the pre-crastination choice reflects automatic execution that requires little deliberation (Fournier et al., 2018; Blinch \& DeWinne, 2019; Ragunath, et al., 2020). For example, Blinch and DeWinne found shorter deliberation times for participants who pre-crastinated relative to those who selected the motorically efficient choice. A more recent study suggested that those with higher working memory capacities may be more likely to deliberate and select the motorically efficient choice (Ragunath, et al., 2020), though it is possible that this relationship reflected the extent to which participants 
(undergraduate college students) exerted maximum effort in completing either task. Notwithstanding, if pre-crastination reflects automatic processing, it is likely due to the fact that the perception of objects activates their affordances, in particular when the objects are relevant for the task at hand (e.g., Jax \& Buxbaum, 2010; Tucker \& Ellis, 1998; see Fournier et al., 2018 for a more extensive discussion). Similarly, the presence of correct affordances for an intended action plan can influence attention (Pavese \& Buxbaum, 2002). Together, these notions suggest that behavioral inhibition is likely required to bypass the objects that are nearer to the participant in order to select the motorically efficient choice (Fournier et al., 2018).

We feel this account holds promise for understanding the choices produced by the bonobos. As noted above, the bonobos typically selected the first object they encountered, either in the context of transporting it alone (pre-crastination) or in the context of selecting both objects. This fits with the notion that bypassing the near object with the correct affordances would require inhibitory control (Fournier et al., 2018). It is also worth noting that chimpanzees, a close relative of the bonobo, have been found to exert inhibitory control in a variety of situations (e.g., Beran et al., 2014; Evans \& Beran, 2007), but the relatively low cost for transporting the tools in our experiment (in which the reward would be the same regardless) likely diminished the inclination to exert more cognitive effort. Correspondingly, given that the bonobos could not be instructed to take only one tool, the selection of the second tool may likewise reflect a lack of inhibition. That said, this explanation might mean the bonobos did not consider the subgoal completed after selecting the first object (which further speaks to the notion that the behavior was automatically executed, particularly for some individuals). Future work investigating the relationship between inhibitory control and pre-crastination would be useful for both humans and bonobos.

The automatic response hypothesis is further supported by Blinch and DeWinne's (2019) observation that the selection of the second object alone appeared to require more deliberation in humans. As we noted above, very few bonobos ever selected this option, and none consistently (unlike the aforementioned human study in which $40 \%$ of human participants consistently selected the motorically efficient choice). Similarly, the notion that correct affordances of the tool may facilitate the triggering of the action plan could explain the results of Phase 2. In Phase 2, the bonobos did not select the first object when it was non-functional. While the objects may have looked superficially similar to the tools, the affordances were clearly different (e.g., the strip of tape relative to the stick). Thus, the role of affordances in triggering the action plan (see Fournier et al., 2018) may have obviated the need for inhibitory control to suppress selection.

Assuming this automaticity theory is correct, we would expect to find pre-crastination behaviors across a wide variety of species. Perhaps the more interesting test, from a cognitive perspective, would be to investigate whether the pre-crastination tendency is reduced as the cost for transport is raised. For example, when Raghunath and colleagues (2020) presented participants with a near cup (that required further transport) that was relatively full of water (and hence required greater attention during transport) versus a far cup that had relatively little water, participants tended to avoid the pre-crastination choice. By not abiding by the automaticity heuristic, the individuals exhibited some flexibility in their choice. Thus, flexibility in choice behavior in the context of these pre-crastination tasks might be a better index of deliberation, as flexibility has also recently been linked with conscious behaviors (Droege \& Braithwaite, 2014; Droege et al., 2021). Planned future experiments will explore pre-crastination using objects that require greater physical effort to move and thus preclude the selection of both objects.

Broadly, our findings are consistent with other studies demonstrating a lengthy evolutionary history for cognitive heuristics (e.g., Chen et al., 2006; Heilbronner \& Hayden, 2016; Krupenye et al., 2015; Shafir et al., 2002) that presumably act to reduce cognitive load during decision making, occasionally at the expense of producing optimal outcomes from an energetic perspective (e.g., Gigerenzer \& Todd, 1999). In a similar vein, research on pre-crastination more generally underscores the need to quantify both cognitive and physical costs associated with behavioral choices (Rosenbaum et al., 2019). On this view, pre-crastination behaviors, which may feel unintuitive on first blush, may accord well with Zipf's principle of least effort (Zipf, 1949). 
We close by noting that understanding the evolutionary roots of pre-crastination is of particular importance given that it is a bias that influences the ordering of action sequences. Similar types of ordering biases (e.g., Easy First) have been posited to play a particularly important role in shaping human behavior, including planning syntactic choices in the context of language production (see MacDonald, 2013). Given the insight that all complex action sequences require a hierarchical plan (Lashley, 1951; see Rosenbaum et al., 2007), finding parallel biases for sequence planning in a closely related primate species may shed new light on the evolutionary origins of some of the most complex forms of human behavior.

Data Availability: Data and coding are available upon request and on GitHub: https://github.com/ngschwob/PRCST Bonobos.

Author Contributions: Natalie Schwob was responsible for experimental design, data collection, data analysis and writing of the manuscript. Amanda Epping was responsible for experimental design and data collection. Jared Taglialatela was responsible for experimental design. Daniel Weiss was responsible for experimental design, data analysis and writing of the manuscript.

Funding: Funding was provided by the Herbert H. Krauss Memorial Graduate Research Endowment in Psychology.

Conflict of Interest: The authors declare no conflicts of interest.

\section{Acknowledgements}

We would like to thank the Herbert H. Krauss Memorial Graduate Research Endowment in Psychology for funding this project. Additional thanks to Renee Margolis for helping code the data and to Chelsea Cox, Emily Cain, Gaila Conklin, Anna Olson, and Calle Uerling for assistance in collecting the data. We would also like to thank David Rosenbaum for contributing to early discussions of the project.

\section{References}

Beran, M. J., Evans, T. A., Paglieri, F., McIntyre, J. M., Addessi, E., \& Hopkins, W. D. (2014). Chimpanzees (Pan troglodytes) can wait, when they choose to: A study with the hybrid delay task. Animal Cognition, 17(2), 197-205.

Blinch, J., \& DeWinne, C. R. (2019). Pre-crastination and procrastination effects occur in a reach-to-grasp task. Experimental Brain Research, 237(5), 1129-1139.

Chen, M. K., Lakshminarayanan, V., \& Santos, L. R. (2006). How basic are behavioral biases? Evidence from capuchin monkey trading behavior. Journal of Political Economy, 114(3), 517-537.

Droege, P., \& Braithwaite, V. A. (2014). A framework for investigating animal consciousness. In G. Lee, J. Illes, \& F. Ohl (Eds.), Ethical Issues in Behavioral Neuroscience. Current Topics in Behavioral Neurosciences, Vol 19 (pp. 79-98). Springer.

Droege, P., Weiss, D. J., Schwob, N., \& Braithwaite, V. (2021). Trace conditioning as a test for animal consciousness: A new approach. Animal Cognition, 24, 1299-1304.

Evans, T. A., \& Beran, M. J. (2007). Chimpanzees use self-distraction to cope with impulsivity. Biology Letters, 3(6), 599-602.

Fournier, L. R., Coder, E., Kogan, C., Raghunath, N., Taddese, E., \& Rosenbaum, D. A. (2018). Which task will we choose first? Precrastination and cognitive load in task ordering. Attention, Perception, \& Psychophysics, 81(2), 489-503.

Fournier, L. R., Stubblefield, A. M., Dyre, B. P., \& Rosenbaum, D. A. (2019). Starting or finishing sooner? Sequencing preferences in object transfer tasks. Psychological Research, 83(8), 1674-1684.

Gigerenzer, G., \& Todd, P. (1999). Fast and frugal heuristics: The adaptive toolbox. In G. Gigerenzer, P. Todd, \& the ABC Group (Eds.), Simple heuristics that make us smart (pp. 11). Oxford University Press.

Heilbronner, S. R., \& Hayden, B. Y. (2016). The description-experience gap in risky choice in nonhuman primates. Psychonomic Bulletin \& Review, 23(2), 593-600.

Hull, C. L. (1943). Principles of behavior (Vol. 422). Appleton-Century-Crofts. 
Jax, S. A., \& Buxbaum, L. J. (2010). Response interference between functional and structural actions linked to the same familiar object. Cognition, 115(2), 350-355.

Kahneman, D., \& Tversky, A. (1979). On the interpretation of intuitive probability: A reply to Jonathan Cohen. Cognition, 7, 409-411.

Krupenye, C., Rosati, A. G., \& Hare, B. (2015). Bonobos and chimpanzees exhibit human-like framing effects. Biology Letters, 11(2), 20140527.

Lashley, K. S. (1951). The problem of serial order in behavior (Vol. 21). Bobbs-Merrill Oxford.

Lebkuecher, A. L., Schwob, N., Kabasa, M., Gussow, A., MacDonald, M. C., \& Weiss, D. J. (under review). Plan reuse in motor and language production. Quarterly Journal of Experimental Psychology.

MacDonald, M. C. (2013). How language production shapes language form and comprehension. Frontiers in Psychology, 4, 226.

Pavese, A., \& Buxbaum, L. J. (2002). Action matters: The role of action plans and object affordances in selection for action. Visual Cognition, 9(4-5), 559-590.

R Core Team. (2016). R: A language and environment for statistical computing. Vienna, Austria. Retrieved from http://www.Rproject.org

Raghunath, N., Fournier, L. R., \& Kogan, C. (2020). Precrastination and individual differences in working memory capacity. Psychological Research, 85, 1970-1985.

Rosenbaum, D. A., Cohen, R. G., Jax, S. A., Weiss, D. J., \& Van Der Wel, R. (2007). The problem of serial order in behavior: Lashley's legacy. Human Movement Science, 26(4), 525-554.

Rosenbaum, D. A., Fournier, L. R., Levy-Tzedek, S., McBride, D. M., Rosenthal, R., Sauerberger, K., VonderHaar, R. L., Wasserman, E. A., \& Zentall, T. R. (2019). Sooner rather than later: Precrastination rather than procrastination. Current Directions in Psychological Science, 28(3), 229-233.

Rosenbaum, D. A., Gong, L., \& Potts, C. A. (2014). Pre-crastination: Hastening subgoal completion at the expense of extra physical effort. Psychological Science, 25(7), 1487-1496.

Rosenbaum, D. A., \& Wasserman, E. A. (2015). Pre-crastination: The opposite of procrastination. Scientific American: Mind Matters. Retrieved from http://www. scientificamerican. com/article/pre-crastination-theopposite-of-procrastination.

Shafir, S., Waite, T. A., \& Smith, B. H. (2002). Context-dependent violations of rational choice in honeybees (Apis mellifera) and gray jays (Perisoreus canadensis). Behavioral Ecology and Sociobiology, 51(2), 180-187.

Tucker, M., \& Ellis, R. (1998). On the relations between seen objects and components of potential actions. Journal of Experimental Psychology: Human perception and performance, 24(3), 830-846.

Valyear, K. F., Fitzpatrick, A. M., \& Dundon, N. M. (2019). Now and then: Hand choice is influenced by recent action history. Psychonomic Bulletin \& Review, 26(1), 305-314.

Wasserman, E. A., \& Brzykcy, S. J. (2015). Pre-crastination in the pigeon. Psychonomic Bulletin \& Review, 22(4), $1130-1134$.

Zentall, T. R., Case, J. P., \& Andrews, D. M. (2018). Procrastination in the pigeon: Can conditioned reinforcement increase the likelihood of human procrastination? Psychonomic Bulletin \& Review, 25(5), 1952-1957.

Zipf, G. K. (1949). Human behavior and the principle of least effort: An introduction to human ecology. AddisonWesley. 